es seien „fraktionsübergreifende“ Vorhaben und Debatten irgendwie edler als kontroverse, etwa indem sie solche als „Sternstunden“ des Bundstages feiern oder erklären, diesmal sei „der Fraktionszwang aufgehoben“. Aber muss dem nicht entgegengetreten werden, statt es zu bekräftigen? Ist es nicht die parlamentarische Kontroverse, das sogenannte „Parteiengezänk“, das eine pluralistische Gesellschaft einigermaßen abzubilden und den Wählern Anhaltspunkte für die je eigene Meinungsbildung zu liefern vermag?

Das Buch scheint letztlich nicht in der Absicht der Aufklärung verfasst zu sein, sondern soll offenbar die Distanz zur parlamentarisch-politischen Wirklichkeit vertiefen. Der Verfasser weiß überall viel besser als die Akteure im Bundestag, was in der Sache richtig ist, was die „Interessen ... des Volkes“ (S. 31) sind und was der angemessene Debattenstil wäre. Er begegnet seinem Gegenstand durchweg und von Anfang an mit der Attitüde des Befremdens. Dem entspricht der Einstieg (S. 5) und der Ausstieg (S. 391): Es geht jedes Mal um „sie und ich“, nämlich die Kanzlerin, Frau Merkel, und der Autor, Herr Willemsen. Sie stehen sich auf Augenhöhe gegenüber, getrennt durch die Glasscheibe des Fernsehgeräts, während ihrer Neujahrsansprachen 2012 und 2013. Nichts hat sich geändert nach dem „Jahr im Parlament“: Ihr Reden bleibt ihm unverständlich, ohne Funktion, betrifft ihn nicht, „doch man kann nicht widersprechen“ (S. 7). So wird der Leser von vornherein darauf eingestimmt, dass Willemsens Beurteilung des Regierungs- und Redestils der Kanzlerin durch seine Eindrücke auf der Bundestagstribüne am Ende bestätigt werden wird - und tatsächlich, so kommt es auch. Wer sich fragt, weshalb der Autor als Einstieg in ein Jahr des Parlaments sich gerade die Regierungschefin als virtuelles Gegenüber gewählt hat, wird entweder vermuten: Darunter tut er es nicht; oder aber: Er findet Regieren eben doch wichtiger und maßgeblicher als Parlieren (vgl. dazu S. 58, wo das Reden sich „vor das Handeln schiebt und es regelrecht unmöglich macht“). Was Neujahrsansprachen und Parlamentsdebatten letztlich zusammenhält, ist die übereinstimmende Bewertung: Es ist immer das Gleiche, das Rituelle, das Uneigentliche und Ungenügende.

Auf dem Klappentext des Buches wird Dieter Hildebrandt zitiert: „Warum ist noch niemand auf diese Idee gekommen?" (nämlich das Plenargeschehen von der Besuchergalerie aus zu beobachten und zu bewerten). Zur Antwort mag ein Diktum von Max Weber beitragen: „Der Einfall ersetzt nicht die Arbeit.“

Wolfgang Zeh

\title{
Pro und contra Parlamentsmacht: viele Fallstricke für empirische Analysen
}

Oberreuter, Heinrich (Hrsg.): Macht und Ohnmacht der Parlamente, Nomos Verlagsgesellschaft, Baden-Baden 2013, 249 Seiten, € 34,-.

Der alten Frage von Macht und Ohnmacht von Parlamenten widmet sich der Sammelband, der zwölf Beiträge vom 10. Passauer Parlamentarismussymposium aus dem Jahre 2011 zusammenträgt. Die meisten Texte behandeln mehr oder weniger prominent den Bundestag, fünf sind einzelnen Länderstudien vorbehalten.

Dass die Machtfrage nur sehr diffizil zu beurteilen ist, wird bei der Lektüre schnell klar. Macht stellt eine grobe Kategorie dar, und Analysen von Einzelaspekten können zu vorschnellen und häufig dem eigentlichen Gegenstand nicht gerecht werdenden Etikettierun- 
gen führen. Werner J. Patzelt geht daher vor allem der Frage nach, woher Parlamentsmacht überhaupt kommen kann. Als zentral identifiziert er die Haushaltsmacht („power of the purse"), die Kommunikationsfähigkeit und den Verantwortlichkeitsmechanismus von Parlamenten. Insbesondere das Haushaltsrecht stellt er als zentrale Machtquelle heraus und leitet dementsprechend als Forderung ab: „Es muss der Regierung unbedingt verwehrt werden, am Parlament vorbei in den Besitz von Finanzmitteln zu gelangen." (S. 45) Er konstatiert zudem einen Machtverlust von nationalen Regierungen und Parlamenten, dem mit einem Aufbau von supranationaler Parlamentsmacht und einem postnationalen Neoparlamentarismus als Analyserichtung begegnet werden sollte.

Geht Patzelt das Thema grundsätzlich an, hat sich Martin Sebaldt für eine detaillierte empirische Analyse entschieden. Er entwickelt einzelne Indikatoren für die Parlamentsfunktionen, die er nach de jure und faktischer Umsetzung anhand einer Zahlenskala „misst“. Eine Erscheinungsweise von Macht, die Patzelt als eine von vier herausgearbeitet hatte, bleibt dabei vollkommen unberücksichtigt, nämlich dass sich Macht auch darüber entfalten kann, dass sie gar nicht ausgeübt werden muss, sondern nur in ihrer Antizipation besteht.

Norbert Lammert geht der Beschneidung von Parlamentsmacht durch die Medien nach: Er kritisiert wie schon des Öfteren die mitunter stiefmütterliche Behandlung des Bundestages durch die Medien, insbesondere durch die gebührenfinanzierten öffentlich-rechtlichen Fernsehsender. Heinrich Oberreuter sieht Positionsverluste von Parlamenten im Entscheidungsprozess, zum Beispiel durch Europäisierung und Medien, betont jedoch, dass Parlamente weiterhin eine "ganz erhebliche“ Rolle (S. 31) spielen, indem sie Legitimität von Entscheidungen, aber auch des gesamten politischen Systems bewirken.

Suzanne S. Schüttemeyer setzt sich mit den häufig äußerst widersprüchlichen Forderungen an Abgeordnete auseinander und fragt, wie die konkreten Repräsentationsbeziehungen von den Bürgern beurteilt werden. Die Ergebnisse einer aktuellen repräsentativen Befragung belegen, dass sie nicht - wie häufig gemeinhin angenommen - generell mit der Arbeit der Parlamentarier unzufrieden sind. Vielmehr beurteilen sie die Arbeit der Abgeordneten im Bundestag besser als deren Arbeit im Wahlkreis.

Hans-Jürgen Papier beklagt, dass die Stellung des Bundestages de jure viel bedeutsamer sei als in der Verfassungsrealität. Er ist der einzige der in diesem Band versammelten Autoren, der uneingeschränkt von Entparlamentarisierung spricht. So kritisiert er etwa Auslagerungen von Entscheidungen in andere Gremien sowie Kooperationen und Vereinbarungen, „auf die sich der Staat einlässt" (S. 84). Auswege sieht er im Mehrheitswahlrecht und in einer Art Selbsttherapie von Parlamenten und Abgeordneten, da sie in Teilen selbst schuld seien an „Macht- und Bedeutungseinbußen“ (S. 87). Das Verhältnis von Bundestag und Bundesverfassungsgericht untersucht Uwe Kranenpohl, vor allem aus der Sicht des Gerichts - durch Äußerungen von jetzigen und ehemaligen Verfassungsrichtern. Er argumentiert, dass vor allem die verfassungskonforme Auslegung durch das Bundesverfassungsgericht Schwierigkeiten bereitet, da hier konkrete Lesarten vorgeschrieben werden und damit den politischen Handelnden der Spielraum eingegrenzt wird. Bemerkenswert ist, dass sich einige der befragten Richter explizit gegen die Lesart wenden, das Gericht werde zum Ersatzgesetzgeber.

$\mathrm{Zu}$ diesen Beiträgen treten fünf länderspezifische Analysen, die von höchst unterschiedlicher Qualität sind. Während Roland Sturm überzeugend herausarbeitet, dass der britische Parlamentarismus „weitaus lebendiger als vielfach angenommen“ (S. 130) ist, gelingt es Josef Braml nicht, die Situation in den USA mit Abstand zu analysieren. Vielmehr liefert er eine Beschreibung aktueller Entwicklungen und geht mehr auf den Präsidenten als auf den Kon- 
gress ein. Sturm verweist darauf, dass das britische Oberhaus ein „parlamentarisches Schwergewicht“ (S. 123) ist, das Regierungen wiederholt empfindliche Niederlagen bescheren kann. Das Unterhaus hat in den letzten Jahren innerinstitutionelle Reformen umgesetzt, die seine Kontrollrechte stärken. Hier bemerkt Sturm zudem „eine bemerkenswerte Tradition der kritischen Selbstreflexion“ (S. 124). Gemeinsam ist beiden Häusern, dass die informelle Vorabinformation und Konsultationssuche durch die Regierenden zugenommen hat.

Adolf Kimmel und Stefan Köppl fragen, wie sich Reformen auf das französische und italienische Parlament ausgewirkt haben. Kimmel geht der Verfassungsänderung von 2008 nach und findet mehr Kontinuitäten als eine neue Parlamentsqualität. Dies liege erstens daran, dass die Reformen zu begrenzt ausfielen, da der rationalisierte Parlamentarismus in Teilen erhalten blieb, und zweitens an der immer noch bestehenden immensen Ämter- und Mandatskumulierung. Damit verbunden ist ein Selbst- und Rollenverständnis der französischen Abgeordneten, das Kimmel verbesserungswürdig erscheint, da so die Gesetzgebung zu Lasten der Kontrollaufgaben überbetont wird. In Italien ging es im Gegensatz zum französischen Fall darum, das Parlament etwas zu zügeln, so mit der Neuausrichtung des Wahlsystems von 1993 und einigen institutionellen Reformen, die die Regierung stärken sollten. Das Problem war und ist, dass sich die in der Logik des parlamentarischen Regierungssystems eigentlich erwartbare Aktionseinheit aus Regierung und der sie tragenden Parlamentsmehrheit in Italien nicht so geschlossen wie nötig zeigt. Dies hat sich laut Köppl auch nach den Reformen nicht geändert, denn das Parlament bleibe weiterhin ein „sehr starker Akteur", da „exekutive Mehrheiten (die die Regierung ins Amt bringen)“ und „legislative Mehrheiten (die die Regierung braucht, um ihre Projekte in Gesetzesform zu bringen“ (S. 194) häufig differieren.

Ellen Bos referiert die Entwicklungen seit 1989/90 in den Staaten Ost- und Mitteleuropas, die zum Teil demokratisch, zum Teil autoritär einzustufen sind. Nachdem die Parlamente in der Umbruchphase von 1990 zentrale Akteure waren, sind ihre Handlungsspielräume seitdem geschrumpft, allerdings in unterschiedlichem Maße. Zudem wurde ihr Alltag im Laufe der Zeit „routinisiert und rationalisiert“ (S. 205). Insgesamt findet Bos starke Parlamente nur in den demokratischen Ländern, die parlamentarische oder parlamentarisch-präsidentielle Systeme haben, während in den autoritären Staaten eher schwache Parlamente anzutreffen sind.

Zur Frage von Macht und Ohnmacht von Parlamenten neigt nur Papier uneingeschränkt letzterem zu. Andere Autoren wie Oberreuter und Patzelt sehen Bedeutungsverluste, betonen aber die (absolute) Notwendigkeit von Parlamenten. Schüttemeyer verweist auf den wichtigen Umstand, dass eine bessere Beziehung zwischen Repräsentierten und Repräsentanten nötig sei, die auch als solche wahrgenommen wird (S. 78). Sebaldt meint, Macht messen zu können, und kommt zu Ergebnissen, die den Befunden anderer Autoren widersprechen, wenn er etwa das britische Parlament an drittletzte Stelle setzt, während Sturm die lebendige Vielfalt des britischen Parlamentarismus würdigt, oder wenn Sebaldt zur Zügelung der Kontrollfunktion aufruft, während beispielsweise Patzelt, Sturm und Kimmel herausarbeiten, wie wichtig diese Funktion ist. Die Länderbeiträge führen zudem vor Augen, dass Machtzustände nicht statisch sind und dass Institutionengefüge immer auch von Reformen am Leben gehalten werden können und müssen. Ein Kritikpunkt, der wiederholt auftaucht, betrifft die Parlamente und Abgeordneten selbst, die von den Autoren aufgerufen werden, sich auch selbst zu engagieren und bestehende Potenziale auszuschöpfen. Diagnostizierte Schwierigkeiten in der Machtfrage werden somit sowohl den Entwicklun- 
gen unter anderem der Medien und von supranationalen Institutionen zugeschrieben als auch ganz prominent den Parlamenten selbst.

In publizistischer Hinsicht ist es schade, dass der Band keinen resümierenden Beitrag enthält, der die Machtfrage anhand der Texte noch einmal reflektiert. Ebenso fehlt ein Verzeichnis der Autoren. Außerdem hätte die redaktionelle Bearbeitung etwas mehr Sorgfalt vertragen, um zum Beispiel fast leere Seiten aufgrund von Tabellenpositionierungen zu vermeiden. Es zeigt sich überdies, dass es Beiträgen in Sammelbänden nicht gut tut, wenn sie auf umfangreichen Monographien (Kranenpohl, Köppl und vor allem Sebaldt) beruhen, da sie so zu Kurzzusammenfassungen werden, ohne einen für diese Art von Text eigentlich notwendigen argumentativen Bogen spannen zu können.

Franziska Carstensen

\section{Seiteneinsteiger im Bundestag: Karriereweg und politische Arbeit}

\section{Bailer, Stefanie, Peter Meißner, Tamaki Ohmura und Peter Selb: Seiteneinsteiger im Deutschen Bundestag, VS Verlag für Sozialwissenschaften, Wiesbaden 2013, 151 Seiten, € 29,99.}

Wer anders ist, erregt Aufsehen. Das gilt natürlich auch in der Politik. Dort werden die so genannten Seiteneinsteiger zumeist kritisch beäugt. Von Quereinsteigern, -denkern und -köpfen ist dann die Rede, von Mutigen, Andersdenkenden und Greenhorns, von parteipolitischen Frischlingen, bunten Vögeln oder Fachleuten ohne parteilichen Stallgeruch: Die Bezeichnungen sind vielfältig. Gerade die Berichterstattung richtet sich häufig auf neue Namen und Köpfe in der Politik. Doch das (mediale) Interesse an Seiteneinsteigern in der Politik war bis vor einigen Jahren deutlich größer als die wissenschaftliche Aufbereitung. Neben dem Politikwissenschaftler Dietrich Herzog, der sich von 1975 an mit politischen Karrieren beschäftigte ${ }^{1}$, sind erst in der jüngeren Vergangenheit einige Werke hinzu gekommen. ${ }^{2}$ Dazu zählt auch die Untersuchung des Autorenteams von der ETH Zürich und der Universität Konstanz, die auf Anregung des Studentenforums des Tönissteiner Kreises und zusammen mit ihm entstand. „Seiteneinsteiger im Deutschen Bundestag“ lautet der Titel, doch das Buch bietet - trotz des geringen Umfangs - mehr: Es ist eine interessante Analyse der Karrierewege aller Bundestagsabgeordneten der 17. Wahlperiode, also von 2009 bis 2013.

Entscheidend für das Gelingen der Studie ist, dass die Autoren die Schwierigkeit erkennen, Seiteneinsteiger in dem heterogenen Feld der Parlamentarier zu identifizieren. Dieser

1 Vgl. unter anderem Dietrich Herzog, Politische Karrieren - Selektion und Professionalisierung politischer Führungsgruppen, Opladen 1975.

2 Speziell mit Seiteneinsteigern in Deutschland haben sich folgende Studien befasst: Robert Lorenz I Matthias Micus (Hrsg.), Seiteneinsteiger. Unkonventionelle Politiker-Karrieren in der Parteiendemokratie. Göttinger Studien zur Parteienforschung, Wiesbaden 2009; Moritz Küpper, Politik kann man lernen. Politische Seiteneinsteiger in Deutschland, Halle (Saale) 2013; allgemeiner zu Politischen Karrieren in Deutschland: Anwar Syed Ali, Karrierewege und Rekrutierungsmuster bei Regierungsmitgliedern auf Bundesebene von 1949-2002, Halle (Saale) 2003; Andreas K. Gruber, Der Weg nach ganz oben. Karriereverläufe deutscher Spitzenpolitiker, Wiesbaden 2009; speziell für Seiteneinsteiger in der österreichischen Politik: Armin Wolf, Promi-Politik. Prominente Quereinsteiger in der österreichischen Politik, Innsbruck / Wien 2005. 